2. If yes, do you have a guideline which describes how these infusions should be prepared?

Where non-standard glucose concentrations were used and a guideline available, NICUs were asked to share this guideline for the purposes of analysis. Following receipt of the guidelines, they were categorised according to the broad method of glucose solution manufacture:

a. Removal of fluid from bag prior to addition of $50 \%$ glucose, taking into account published overage.

b. Removal of fluid from bag prior to addition of 50\% glucose, not taking into account published overage.

c. Addition of $50 \%$ glucose, without prior removal of fluid from bag.

d. Mixing ratios of concentrations in a burette.

e. 'Piggybacking' a 50\% glucose infusion onto an infusion of $5 \%$ glucose, guided by use of an online calculator.

Results $69.2 \%$ of the 65 NICUs contacted responded $(n=45)$. $66.7 \%$ of respondents $(n=30)$ had guidelines in use: these 30 guidelines were subjected to analysis.

Method a) was used in $6.7 \%$ of guidelines seen $(n=2)$; method b) was used in $60 \%$ of cases $(n=18)$; method c) was used in $3.3 \%$ of cases $(n=1)$; method d) was used in $6.7 \%$ of cases $(n=2)$; method e) was used in $10 \%$ of cases $(n=3) .6 .7 \%$ of guidelines used a different method according to the glucose concentration required $(n=2) .6 .7 \%$ of guidelines advised preparation of glucose in a syringe rather than an infusion bag $(n=2)$.

Although method b) was the most commonly used, there was wide variation in recommended volumes to be added and/or removed.

Only $6.7 \%$ of guidelines reviewed specified the brand of infusion bag to be used $(n=2)$.

Conclusions Considerable variation was seen in the methods of glucose infusion preparation used throughout the UK, suggesting a range of opinions as to the most accurate method of manufacture. Further work is needed to determine the relative accuracy of the different methods, and the clinical significance of the variation observed.

\section{P03 PREPARING GLUCOSE INFUSIONS IN NEONATAL INTENSIVE CARE: DOES IT MATTER WHICH METHOD IS USED?}

${ }^{1}$ Adriece Al Rifai*, ${ }^{2}$ Robyn Hart, ${ }^{1}$ Andrew Wignell. ${ }^{1}$ Nottingham University Hospitals NHS Trust; ${ }^{2}$ School of Pharmacy, University of Nottingham

10.1136/archdischild-2020-NPPG. 12

Aims Administering intravenous (IV) glucose is common on the Neonatal Intensive Care Unit. Bedside preparation of glucose solutions is often necessary, usually through addition of concentrated $50 \%$ glucose to a commercially available bag. Accuracy in the glucose concentration of locally prepared bags will be influenced by a number of factors: variable overages in IV fluid bags, method of preparation and imprecision of measurement during preparation. We aimed to assess the accuracy of three different methods of preparation which had been identified through a national survey.

Methods Bags of $12.5 \%, 15 \%$ and $25 \%$ glucose were manufactured through the addition of $50 \%$ glucose solution to commercially available bags of $10 \%$ or $20 \%$ glucose. Three bags of each concentration, were manufactured by each of the methods below: a. Removal of fluid from base bag prior to addition of $50 \%$ glucose, taking into account published overage.

b. Removal of fluid from base bag prior to addition of $50 \%$ glucose, not taking into account published overage.

c. Addition of $50 \%$ glucose, without prior removal of fluid from base bag.

Three $5 \mathrm{~mL}$ samples were then taken from each prepared bag and sent for analysis. Glucose concentration was measured using a quantitative spectrophotometric method. As a control, three $5 \mathrm{~mL}$ samples were taken from three bags each of commercially available 5\%, $10 \%$ and $20 \%$ glucose infusion solutions and assayed as above.

Results A total of 81 'test' samples were sent for analysis along with 27 control samples. One 20\% glucose control sample was lost in transport meaning that 80 samples were analysed. The median result for each concentration and method was calculated. For method a) where the intended final glucose concentration was $12.5 \%, 15 \%$ and $25 \%$, the actual concentrations obtained were $11.2 \%, 13.3 \%$ and $22.9 \%$ respectively. For method b) where the intended final glucose concentration was $12.5 \%, 15 \%$ and $25 \%$, the actual concentrations obtained were $12.4 \%, 13.4 \%$ and $22.0 \%$ respectively. For method c) where the intended final glucose concentration was $12.5 \%, 15 \%$ and $25 \%$, the actual concentrations obtained were $12.1 \%, 13.8 \%$ and $20.3 \%$ respectively. For the $5 \%$, $10 \%$ and $20 \%$ control solutions the median reported glucose concentrations were $5.1 \%, 10.3 \%$ and $19.9 \%$ respectively.

Conclusions Irrespective of method used and the intended strength, the measured glucose concentration was lower than that being aimed for. In some cases, the glucose concentration was only $80 \%$ of that intended. It is not possible to conclude that one method is superior in terms of accuracy. Although it might be possible from our results to suggest the most accurate method for each concentration, this is unlikely to be predictable as manufacturers quote overages as a range rather than an absolute value. In clinical practice, preparation of a glucose solution with a lower concentration than that expected may result in prolonged hypoglycaemia with potential neurological sequelae. An alternative to bedside manufacture of glucose infusion solutions is needed. This could include pharmacy compounding of glucose strengths not commercially available or 'piggy-backing' of 50\% glucose onto an infusion of a commercially available strength, ideally supported by a glucose load calculator.

\section{P04 BARRIERS AND FACILITATORS TO MEDICINES ADHERENCE IN CHILDREN: A SYSTEMATIC REVIEW}

Mohammed Aldosari* ${ }^{\star}$ Ana Oliveira, Sharon Conroy. University of Nottingham

\subsection{6/archdischild-2020-NPPG.13}

Aim Improving adherence to medicines in children with chronic conditions may lead to significant economic and health benefits. ${ }^{1}$ To improve adherence, the multifactorial causes of poor adherence should be understood. ${ }^{1}$ A systematic review for barriers and facilitators to medicines adherence in children was conducted seven years ago. ${ }^{2}$ We updated this to identify barriers and facilitators to medicines adherence in children reported in the last ten years.

Method A systematic literature search was performed using PubMed, EMBASE, Medline, CINAHL, IPA and Cochrane library databases covering the period November 2008 to 
March 2019. Inclusion criteria were original research studies identifying barriers and/or facilitators of medicines adherence in children (aged 0-18 years) and included all countries and languages. Exclusion criteria included review articles, editorials, conference papers, reports and studies in adults only. As a reliability measure, $5 \%$ of titles and abstracts were assessed independently by a second researcher. Quality assessment was performed on all included studies using the STROBE checklist for observational studies and Cochrane collaboration tools for randomised controlled studies and was checked by a second researcher.

Results Of 9,360 papers identified by the search, only 172 articles met the inclusion criteria. Most studies were conducted in the US (76), with 11 in the UK, six in Canada and the remaining 79 studies in various countries. Diseases studied included: HIV/AIDS (60), asthma (25), kidney or liver diseases and transplants (18), psychiatric disorders (12), inflammatory bowel disease (10), epilepsy (9) and others (38). Various tools were used to identify barriers and facilitators to medicines adherence. These included 131 studies which used individually designed questionnaires, 32 studies used validated questionnaires and the remaining 9 studies used patients' medical data. Forgetfulness and fear of side effects were the most common reported barriers to medicines adherence. Others reported barriers to adherence included family conflict, weak patient-provider relationships, stigma and discrimination, drug regimen complexity and lack of support from families. Factors reported to facilitate high rates of adherence included the linking of medicine taking with daily life routines, using reminders to avoid forgetfulness, a higher level of caregivers and parental education and good communication between healthcare professionals, patients and parents.

Conclusion The main findings of this systematic review show that children faced many and varied barriers to medicines adherence with different diseases. Using reminders to avoid forgetfulness and good communication between healthcare professionals, patients and parents were the most common facilitators. To achieve optimal adherence, healthcare providers need to be aware of these barriers and to consider the most appropriate facilitators to encourage patients to take their medicines as prescribed.

\section{REFERENCES}

1. Brown MT, Bussell JK. Medication adherence: WHO cares? Mayo Clin Proc 2011:86:304-14

2. Elliott RA, Watmough DE, Gray NJ, Conroy S, Lakhanpaul M, Pandya $H$, et al. Talking about medicines (TABS): a multi-method study to understand reasons for medicines non-adherence in children and young people with chronic illness, and to improve their contribution to managing their medicines. Natl Inst Heal Res 2012; 1-423.

\section{P05 AN AUDIT OF PROTON PUMP INHIBITOR PRESCRIBING, IN ADHERENCE WITH THE TRUST'S GUIDANCE, IN GASTRO-OESOPHAGEAL REFLUX DISEASE}

${ }^{1}$ Yusuf Asif, ${ }^{2}$ Chi Huynh* ${ }^{2}$ Awais Hussain. 'Birmingham Women's and Children's NHS Foundation Trust; ${ }^{2}$ Aston University, Birmingham

10.1136/archdischild-2020-NPPG.14

Aim The effectiveness of proton pump inhibitors (PPIs) have been demonstrated. Nevertheless, the choice of PPI that should be used is less absolute. The clinical effectiveness, availability of the formulation, co-morbidities, route of administration and lowest acquisition cost are all considerations that should be accounted for when determining the appropriate therapy. Current Trust guidance recommends lansoprazole capsules and oral dispersible tablets as the first line PPI, unless other indications preclude its use. Other UK hospitals have audited PPI prescribing and their findings highlight that adherence upon deployment was poor. ${ }^{12}$ This audit aims to assess if written outpatient prescriptions are adhering to the guidelines.

Method This study was conducted prospectively in the outpatient pharmacy, between February and March 2019. The defined data collection period was 5 weeks, which included a 1 -week pilot study. The accumulation of data involved reviewing all outpatient prescriptions, whereby PPIs were prescribed, noting if the Trust's guidance on PPI prescribing was being adhered to. Data was collected via a structured pro forma to assess the percentage compliance against the three predetermined standards:

Standard 1 - Is the PPI prescribed appropriate?

Standard 2 - Is there a documented indication for the prescribed PPI?

Standard 3 - Is the dose appropriate for the patient?

Results There were a total of 84 prescriptions received from 13 different specialties. The age range of patients was 1 month to 16 years with a mean $( \pm$ median) age of $7.66 \pm 7$ years. The overall compliance with the Trust's guidelines for standards 1, 2 and 3 were $76 \%, 88 \%$ and $100 \%$ respectively. The infant and toddler age group ( 28 days - 23 months) showed the least compliance in standard 1, the choice of appropriate PPI (63\%). The most common indication was gastro oesophageal reflux disease. Paediatric Gastroenterology received the greatest number of prescriptions over the data collection period. $12 \%$ of prescriptions did not have a documented indication and the most common PPI prescribed in the outpatient pharmacy was lansoprazole, which accounted for $64(77 \%)$ of the prescriptions.

Conclusion The findings in this study are synonymous to that of other audits conducted in UK hospitals, where compliance with PPI guidelines were explored. Possible factors that could be attributed to the low levels of adherence are problems with implementation, lack of enforcement of the guidelines, patient/guardian preferences and presence of enteral feeding tubes. Clinicians should monitor their prescribing and where applicable, switch patients who are currently on omeprazole suspension, to lansoprazole oral dispersible tablets/capsules. This could lead to significant monetary savings for the Trust.

\section{REFERENCES}

1. Derbyshire Joint Area Prescribing Committee. Gastro-oesophageal reflux disease: recognition, diagnosis and management in children and young people. 2015

2. Pan Mersey Area Prescribing Committee. Pharmacological management of gastrooesophageal reflux disease (GORD) in children and young people in primary and secondary care. 2016.

\section{P06 IMPROVING RENAL FUNCTION MONITORING DURING CHEMOTHERAPY- A ROLE FOR CYSTATIN C?}

Rachel Boys*. Leeds Teaching Hospital NHS Trust

\subsection{6/archdischild-2020-NPPG.15}

Aim Renal toxicity causes major morbidity following chemotherapy- abnormal iGFRs may be detected in up to $73.7 \%$ of patients. ${ }^{1}$ Creatinine is universally used as a biomarker to track fluctuating function and to calculate surrogate glomerular filtration rate (GFR) in the form of estimating 